Erschienen in: Oskar Becker im phänomenologischen Kontext / Sattler, Jochen (Hrsg.). - Paderborn: Wilhelm Fink, 2020. - (Neuzeit und Gegenwart). - S. 75-86. - ISBN 978-3-7705-6465-1

\section{Gab es eine geschriebene ungeschriebene Lehre Platons? Oskar Beckers Rekonstruktion des 2. Teils des Parmenides}

\author{
1. Vorbemerkung
}

Es ist ungefähr zwanzig Jahre her, als mit der tatkräftigen Unterstützung von Jürgen Mittelstraß die Gründung eines philosophischen Archivs an der Universität Konstanz in Angriff genommen wurde. Die ersten Archivalien waren eine Kopie der wissenschaftlich relevanten Teile des Nachlasses von Hugo Dingler, meine eigene Sammlung von Kopien aus dem Nachlass Ernst Machs, sowie ein kleines Päckchen mit dem, was Mittelstraß mir als „Nachlass Oskar Becker“ überreichte.

Bis allerdings der wirkliche Nachlass Oskar Becker im Juni 1999 in Konstanz eintraf, sollten noch viele Jahre vergehen. Inzwischen war das „Philosophische Archiv an der Universität Konstanz" zum größten Archiv seiner Art in Europa herangewachsen.

Was war nun in jenem braunen Päckchen mit der Aufschrift „Nachlass Oskar Becker"? - Nun, es war nicht der Nachlass Becker, wohl aber eine von Becker selbst noch zur Publikation zusammengestellte Aufsatzsammlung, die bis auf eine Ausnahme aus Sonderdrucken der jeweiligen Erstpublikationen bestand. ${ }^{2}$ Von dieser Ausnahme, dem Nachlasskonvolut mit dem rätselhaften Titel „Über

Wiederabdruck (mit freundlicher Genehmigung des Verlags Walter de Gruyter) aus Gereon Wolters/Martin Carrier (Hg.), Homo sapiens und Homo faber: Epistemische und technische Rationalität in Antike und Gegenwart. Festschrift für Jürgen Mittelstraß, Berlin/New York (de Gruyter) 2005, 51-63.

' Vgl dazu Wolters/Schroeder (1979)

${ }^{2}$ Ursprünglich scheint Becker eine Publikation seiner "Wissenschaftlichen Abhandlungen" aus verschiedenen Gebieten geplant zu haben. Die Unterabteilungen waren „A. Zur Mathematikgeschichte, B. Zu den Grundlagen der Mathematik, C. Philologica, D. Philosophica, E. Zur Logik der Modalitäten, F. Varia“" (OB 6-2-3). Dieses umfassende Projekt hatte sich offenbar nicht realisieren lassen. Die meisten der für die Abteilung „Philosophica“ vorgesehenen Arbeiten erschienen als Becker (1963). - Das erwähnte Konvolut „Nachlass Becker“ war für eine

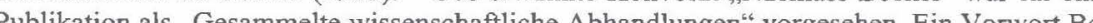
Publikation als "Gesammelte Die Siglen beziehen sich auf den Nachlass Oskar Becker im „Philosophischen Archiv der Universität Konstanz". Ich danke der Archivarin, Dr. Brigitte Parakenings, fuir ihre wie immer tatversität Konstanz“". Ich danke der Archivarin, Dr. Brigitte Parakenings, für ihre wie immer tat-
kräftige Unterstützung. - Mein ganz besonderer Dank gilt Emidio Spinelli (Rom), einem auskräftige Unterstützung. - Mein ganz besonderer Dank gilt Emidio Spinelli (Rom), einem aushätte, mich zu einem so schwierigen Thema ausserhalb meiner engeren Arbeitsgebiete zu äus- 
den Platonischen Dialog Parmenides“ mit dem rätselhaften Untertitel: „Urfassung und metaphysische Deutung" (Signatur: OB 6), soll im Folgenden die Rede sein. Er besteht aus drei Teilen. Der erste Teil ist der eigentliche Aufsatz und besteht aus zwei Abschnitten: „I. Metaphysische Deutung des zweiten Teils des platonischen Dialogs >Parmenides<" und "II. Die Urfassung des zweiten Teils des platonischen Dialogs $>$ Parmenides $، " 3$ Den beiden übrigen Teilen werden wir uns ganz zum Schluss zuwenden.

\section{Parmenides-Probleme}

Platons Parmenides gilt als ein sehr schwieriger Dialog. Die Probleme, die er bietet, lassen sich in zwei Gruppen einordnen: inhaltliche und formal-literarische.

Bevor wir uns hier Einzelheiten zuwenden, ein Wort zur Chronologie - zur fiktiven, d.h. in den Dialogen selbst inszenierten, wie auch zur realen, d.h. die tatsächliche Reihenfolge betreffend, - in der Platon seine Dialoge geschrieben tatsächliche Reihenfolge betreffend, - in der Platon seine Dialoge geschrieben hat. In der fiktiven Che man in frühe, mittlere und In der realen Chronologie sich beim Parmenides jedoch um ein Spätwerk, wohl kurz nach -370 geschrieben ${ }^{5}$, dem, ,insbesondere Phaidon, Symposion und Republik voraus" gingen und dem - durch Bezugnahmen gesichert - u.a. Theaitet, Sophistes und Politikos nachfolgen (von Kutschera, 1995, S. 1), wobei sich freilich in dieser Abfolge keine Entwicklung des platonischen Denkens zeigt. Der Parmenides bildet vielmehr mit den drei auf ihn folgenden Dialogen eine kompositorische Einheit (Guthrie, Bd. V, 1978, S. 33).

Die formalen Probleme haben vór allem damit zu tun, dass der Dialog aus zwei auf den ersten Blick sehr heterogenen Teilen besteht. Im ersten Teil wird über ein Gespräch berichtet, das der „sehr junge“" (127c5f.) Sokrates u.a. mit dem alten Parmenides und dessen Schüler Zenon geführt habe. $\mathrm{Zu}$ dessen $\mathrm{Be}-$ sonderheiten gehört, dass Parmenides dem Sokrates, nachdem dieser mit einer Befragung Zenons begonnen hatte, bald einmal die Gesprächsführung aus der Hand nimmt. Er macht Sokrates in der Folge auf eine Reihe von Paradoxien aufmerksam, die mit dessen Auffassung von Ideen verbunden sind. ${ }^{6}$ Der junge

${ }^{3}$ Die Paginierung der beiden Teile ist nicht einheitlich, da sie aus einem grösseren Zusammenhang Die Paginienten werste Abshnitt des ersten Teils als Al Seitenzahl zitiert, der zweite als AII + Seitenzahl. Die beiden übrigen Teile mit B bzw. C.

Seiter Chronologie naturgemäss der letzte.

Dies ist die Auffassung von Brisson, 1994, S. 14.

${ }^{6}$ Ich vermeide das Wort "Ideenlehre". Damit folge ich der wegweisenden Platon-Studie von Wolfgang Wieland: „Eine Ideenlehre, die diesen Namen verdiente und die Aussagen über den logischen, den ontologischen und den axiologischen Status der Idee in systematischem Zusammenhang begründete, sucht man im Platontext jedoch vergebens. Selbst ein griechisches Äquivalen
Sokrates aber kann diese Paradoxien nicht auflösen und bekennt seine Ratlosigkeit (135 c7). Parmenides hält ihm schließlich vor, dass er zu früh, d.h. vor gehöriger Übung ( $\pi \rho i v \gamma v \mu \nu \alpha \sigma \theta \tilde{\eta} v \alpha$ ) ), sich über die Ideen ausgelassen habe (135 c8). Allseits dazu aufgefordert, erklärt sich Parmenides schließlich bereit, eine Probe solcher logisch-dialektischer „Gymnastik“ zu liefern - und eben darin besteht der zweite Teil des Dialogs. Erfolgreiches Absolvieren der „Gymnastik“ soll - so scheint es Becker zu sehen - die für die Meisterung der Ideenkonzeption erforderlichen Kompetenzen bereitstellen oder doch wenigstens üben.

Über den Zusammenhang bzw. Nicht-Zusammenhang der beiden Teile und damit über den systematischen Stellenwert der Intellektualgymnastik des zweiten ist - ohne schlüssiges Resultat - in der Forschung viel gerätselt worden. Der gerade skizzierte erste zeigt den für viele Dialoge uns vertrauten, lebhaften, humorvollen und sorgsam inszenierten Austausch von Argumenten. Der zweite hingegen „consists of almost thirty Stephanus pages of unadorned argument. Yet when deciding what, exactly, the character of each part is and how they fit together to make up a whole, scholars still express doubts" (Meinwald, 1991, S. 3). Wie sollen diese beiden literarisch so disparaten Teile eine Einheit bilden?

Aber auch hinsichtlich des Inhalts gibt es viele Meinungen. Kutschera (1995, S. 12) stellt, nach einer Auflistung der wichtigsten Interpretationsansätze lakonisch fest: „Soweit ich sehe, gibt es bisher keine wirklich befriedigende Interpretation des Parmenides"?

Als Becker an der Jahreswende 1942/43 seinen Parmenides-Artikel verfasste,${ }^{8}$ war die Lage nicht viel anders. Den Anstoß zu seiner Arbeit hatte die Parmenides-Interpretation von Max Wundt gegeben (Wundt, 1935). ${ }^{9}$ Becker schliesst sich ,im Großen und Ganzen“ (AI, S. 1) der Wundtschen ,metaphysischen" Deutung des Parmenides an und beschränkt seine Überlegungen - wie auch Wundt selbst - auf den zweiten Teil des Dialogs. Die „,metaphysische Deutung" des Parmenides verfolgt - damals wie heute ${ }^{10}$ - bei allen Unterschieden im Einzelnen eine Interpretationslinie, die von den Neuplatonikern begründet wurde und in der ontologisch-dialektischen Deutung Hegels ihren vorläufigen Höhepunkt erreichte. Sie besteht darin, „die grossartige Dialektik im zweiten Teil des Parmenides wirklich in ihrer Tiefe, als Ausdruck der wahren metaphysischen Überzeugungen Platons" zu deuten (Wundt, 1935, S. 27). ${ }^{11}$

für den uns so vertrauten Ausdruck, Ideenlehre' fehlt im Bereich der unzweifelhaft echten Schriften Platons" (Wieland, 1999, S. 96). Über die vielfach neu gesehene Stellung der Ideenkonzeption orientieren u.a. Gonzalez (Hrsg.), 1995 und Vegetti, 2003.

${ }^{7}$ Hierin stimmen ihm vermutlich alle Interpreten bei

${ }^{8}$ Das Manuskript trägt die Aufschrift „Bonn, Dez. 1942 - Jan. 1943“.

"Max Wundt war im Übrigen, aufbauend auf einer frühen „völkischen“ Orientierung, - ohne Parteimitglied zu sein - einer der prononciertesten Vertreter einer Naziphilosophie. Als Naziphilosoph ist (wenigstens zeitweise) auch Becker einzustufen. Vgl. Wolters, 2002.

${ }^{10}$ Eine neuere Vertreterin der metaphysischen Deutung ist I. Schudoma. Sie selbst spricht von ,ontologisch-systematische(r) Interpretation“ (z.B. Schudoma, 2001, S. 10).

Die wichtigste Alternative zur metaphysischen Deutung in ihren unterschiedlichen Varianten ist die logische Deutung, vgl. z.B. von Kutschera, 1995. 
Becker, dessen eigene Version der metaphysischen Deutung im nächsten Abschnitt behandelt wird, sieht jedoch ,die Entwicklung seines metaphysischen Systems nicht als die einzige Aufgabe an [...], die sich Platon im Parmenides stellt. Auch die Durcharbeitung der Dialektik des Einen ist Selbstzweck, in Verbindung mit der Weiterführung und schliesslichen Selbstaufhebung des eleatischen Ansatzes, wie sie im Sophistes dann vollendet wird" (AI, S. 24).

Was nun die Form des Parmenides betrifft, vertritt Becker mit nicht geringem Aufwand eine überraschende, um nicht zu sagen kühne These: der zweite Teil des Parmenides war ursprünglich ein nicht dialogisierter, interner Lehrtext der Akademie, dem Platon dann eine, allerdings nur oberflächliche Dialogform gegeben und - etwas gewaltsam - mit dem ersten Teil verbunden hat. In Abschnitt IV wird Genaueres berichtet.

\section{Beckers metaphysische Deutung}

Der zweite Teil des Parmenides besteht aus acht so genannten „Hypothesen“ aus denen dann Schlussfolgerungen gezogen werden. Der Aufbau des Ganzen ist streng symmetrisch. Platon (oder Parmenides) geht aus von zwei Basishypo-

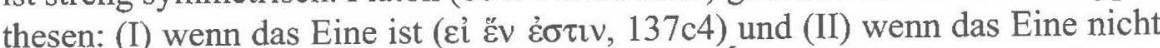

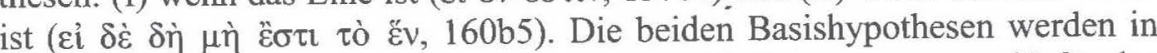
einem ersten Schritt je zweifach aufgefächert: (1) Folgerungen aus (I) für das Eine (A); (2) Folgerungen aus (I) für vom Eins verschiedenes Andere (B). Entsprechend für (II): (1) Folgerungen aus (II) für das Eine; (2) Folgerungen aus (II) für vom Einen verschiedenes Andere. Schließlich werden diese vier „Hypothesen" je zweifach aufgefächert und zwar so, dass in jedem der vier Fälle untersucht wird, was sich ergibt, wenn dem Einen bzw. dem Anderen alle Bestimmungen zu- oder abgesprochen werden. Das ergibt insgesamt die acht „Hypothesen", vier ,positive" und vier ,negative", irgendwie ,subjektive“", die nach Becker einander entsprechen müssen.

Im Dialog folgen sie so aufeinander: ${ }^{13}$

\section{I. wenn das Eine ist:}

A 1: Erste Folgerung für das Eine

A 2: Zweite Folgerung für das Eine

$B$ 1: Erste Folgerung für das Andere

B 2: Zweite Folgerung für das Andere

1. Hypothese

2. Hypothese

3. Hypothese

4. Hypothese

${ }^{12}$ Die Forschung ist sich im Übrigen uneins darüber, ob acht oder neun „Hypothesen“ erörter werden. Becket schliesst sich Wundts (1935, S. 26, S. 41 ff.) Auffassung an, dass es acht seien. Er tut dies allerdings weniger aus historisch-philologischen als systematisch-logischen Gründen.

13 nach dem dem Schema bei Wundt (1935, S. 29).

II. Wenn das Eine nicht ist

A 1: Erste Folgerung für das Eine

A 2: Zweite Folgerung für das Eine

B 1: Erste Folgerung für das Andere

5. Hypothese

6. Hypothese

7. Hypothese

B 2: Zweite Folgerung für das Andere

8. Hypothese

Die metaphysische Deutung verlangt nun, dass den „positiven“ Hypothesen (d.h. wenn das Eine ist) Stufen des Seins entsprechen. Becker schliesst sich hier mit Wundt den spätantiken, neuplatonisch orientierten Kommentatoren an: $a b-$ solut Eines - Idee - materielle Dinge - Materie sind die entsprechenden Seinsstufen. ${ }^{14}$ Schwieriger sind die Zuweisungen zu den ,negativen“ Hypothesen. Bezüglich der fünften und sechsten folgt Becker Wundt. In der fünften wird

„wieder die Idee dar(gestellt), aber in negativer Hinsicht - als nichtseiende oder andere, d.h. im Gegensatz gegen andere Ideen stehende. Das Eine im Sinne der zweiten Hypothese (das ع̈v őv) also bezüglich gesetzt, ist nichtseiend (abwesend), genauer nicht so seiend wie andere Einheiten". [...] Die 6. Hypothese zielt dagegen auf das unbezügliche (absolute oder höchste) Eine, ebenfalls als nichtseiendes (abwesendes), also negativ betrachtet. Im Ergebnis unterscheidet sie sich nicht von der 1. Hypothese, da das höchste, transzendente' Eine über den Gegensatz von Sein und Nichtsein hinaus ist - bekanntlich ein westlicher und östlicher Mystik gemeinsamer Satz" (AI, S. 25).

Was hier auffällt ist, dass die fünfte, die erste „negative“ Hypothese nicht etwa der ersten „positiven“ entspricht, sondern der zweiten. Entsprechend besteht eine Zuordnung der zweiten ,negativen“ zur ersten ,positiven“ Hypothese. ${ }^{15}$

Die 7. Hypothese ist „das genaue Gegenstück zur Dritten“ (AI, S. 26) und auf die Erscheinungen, sowohl ,ihre Erscheinungsweisen in der Wahrnehmung“, aber auch - gegen Wundt - ,Spiegelbilder und Schatten, perspektivische Gemälde, optische Täuschungen, Traumphantasien und Gespenster, [...]

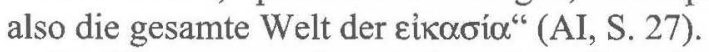

Zur Bestimmung der mit der achten Hypothese verbundenen Seinsstufe verwendet Becker, einer der bedeutendsten Erforscher der antiken Proportionenlehre, eine Proportionalüberlegung. Danach sollten ,,bei der Neigung Platons zu analogischem architektonischen Aufbau" (AI, S. 26f.) folgende Proportionen bestehen:

${ }^{14}$ Becker fügt (AI, 25) in Klammern hinzu; , „die dem Raum nahe steht“. Er bezieht sich damit offenbar auf Platons Theorie der $\chi \hat{\omega} \rho \alpha$ im Timaios (48e2ff.). Hier wird die $\chi \dot{\omega} \rho \alpha$ als dritte Gattung" (

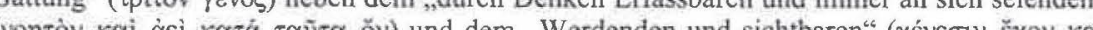

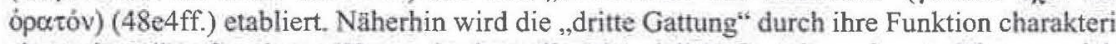
siert: ein selbst formloses Wesen, in dem alle Materialität Gestalt annimmt. (Platon spricht

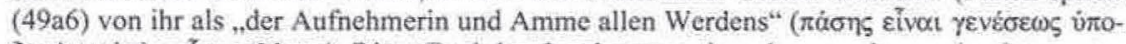

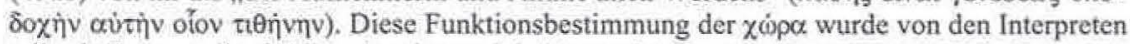
teils als Raum, teils als Materie oder auch beides gedeutet.

${ }^{15}$ Becker gibt dafür eine ausführliche Begründung, die hier nicht dargestellt werden kann. 
(1) 3. Hypothese : 4. Hypothese $=7$. Hypothese : 8 . Hypothese (2) 3 . Hypothese : 7. Hypothese $=4$. Hypothese : 8 . Hypothese

Wenn man nun ,den noch unbekannten Gegenstand der 8. Hypothese mit X bezeichnet" (AI, S. 27) ergibt sich:

(1) Ding : Materie = Erscheinung : X

(2) Ding : Erscheinung = Materie : $X$

Becker resümiert:

„Nach der ersten Proportion ist also X die Materie der Erscheinung, Shakespeares "Stoff aus dem die Träume gemacht sind" (,Der Sturm“, IV. Akt, 1. Scene), nach

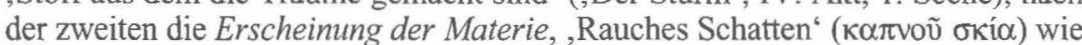
das griechische Sprichwort sagt. Das ist aber in beiden Fällen eine Beschreibung des Nichts. Denn Phantasmen, bloße Erscheinungen sind stofflos (vgl. Aristoteles, de anima III, 8; p. 432a9) und andererseits gibt es keine Erscheinung der letzten Materie, da diese dem Raume gleichzusetzen ist. Denn der leere Raum ist für sich nicht wahmehmbar, ist kein Phänomen oder Phantasma."

Becker fügt noch die interessante Beobachtung an, dass „die mathematischen Gebilde (worunter auch die idealen Himmelskörper und musikalischen Töne zu verstehen sind) hier im Parmenides nicht als besondere Seinsstufe auftreten."

\section{Platons geschriebene, ungeschriebene Lehre}

Was den inhaltlichen Zusammenhang der beiden Teile betrifft, ist Becker, obwohl er viele Bezüge sieht, skeptisch: ,im ganzen ist doch im zweiten Teil keine befriedigende Lösung der Problematik des ersten zu erblicken" (AII, S. 2). ${ }^{17}$ Eine Reihe von weiteren, in der Literatur längst benannten Gründen haben es sodann für Becker „wahrscheinlich gemacht, dass der zweite Teil zunächst selbständig war" (AII, S. 3). Wenn man nun weiterhin bedenke, dass dem zweiten Teil die Dialogform irgendwie künstlich auferlegt zu sein scheine, stelle sich die Frage nach der Möglichkeit,

,diesen zweiten Teil in seiner ursprünglichen Fassung, als eigene ,Schrift ${ }^{6}[\ldots]$ wiederherzustellen. Wenn es sich nur um ein blosses Scheingespräch handelt, ist dann nicht der dialogischen Fassung eine andere in zusammenhängender Rede dann nicht der dialogischen Fassung eine andere in zusammenhängender Rede
vorausgegangen? Und hätten wir nicht, wenn eine solche Wiederherstellung der Urfassung gelänge, ein Beispiel einer echten platonischen Lehrschrift vor uns,

${ }^{16}$ In einer überzeugenden Auslegung des so genannten Liniengleichnisses in Platons Staat komm Wieland $(1999,201 \mathrm{ff}$.) zum gleichen Resultat: „In der Gedankenwelt des Liniengleichnisses ist für die Annahme spezifisch mathematischer Gegenstände kein Platz.“ - Diese Auffassung teilt Becker für das Liniengleichnis jedoch nicht, wie sich aus einem dann doch nicht für die Veröffentlichung vorgesehenen Teil des ursprünglichen Manuskripts ergibt (OB 1-1c-7, S. 37).

Eine solche beansprucht wiederum u.a. von Kutschera, 1995, S. $137 \mathrm{ff}$. für seine auf der mereologischen Logik beruhende logische Deutung des Parmenides. eine für den inneren Kreis der Akademie gedachte Belehrung oder Vorlesung? So wissen wir ja auch von den Ausführungen Platons ,Über das Gute', die mehrfach (u.a. von Aristoteles) nachgeschrieben wurde, aber bis auf wenige, nicht einmal wörtlich überlieferte Fragmente verloren gingen. [...] niemand ist anscheinend bisher auf den Gedanken gekommen, eine solche Lehrschrift aus dem Material der platonischen Dialoge selbst wiederzugewinnen."

Mit diesen Worten betritt Becker - möglicherweise ohne es bemerkt zu haben ${ }^{18}$ - ein vermintes Gelände: das Feld der, ,ungeschriebenen Lehre“ Platons. Becker wird nämlich von Verfechtern einer dem inneren Kreis der platonischen Akademie vorbehaltenen „esoterischen Sonderlehre Platons“ gelegentlich zu den Vorläufern der so genannten Tübinger Schule (insbesondere Konrad Gaiser und Hans Joachim Krämer) gezählt (Gaiser, 1968, S. 21).

Jürgen Mittelstraß gehört zu den pointiertesten Kritikern dieses Ansatzes, der unter anderem eine, inhaltliche Eigenart und (den) Vorrang der esoterischen Lehre Platons" impliziert (Krämer, 1967, S. 23). ${ }^{19}$ Speziell mit Blick auf Krämer bemerkt Mittelstraß, dass dieser ,in einer sorgfältigen Rekonstruktion der Systementwürfe Speusipps [...] und Xenokrates' [...] den innerakademischen Ursprung neuplatonischer Lehren nachgewiesen" habe,

"wobei nun gerade diese Rekonstruktion, wenngleich auch vom Autor selbst so sicherlich nicht beabsichtigt, zeigt, in welch phantastischen Spekulationen sich platonisches Denken alsbald verliert. Besonders deutlich wird diese Entwicklung wieder in dem Versuch einer theoretischen Fundierung der praktischen Philosophie [...], der nunmehr anscheinend konkurrenzlos das akademische Denken beherrscht. Man wird deshalb auch Krämers [und Gaisers] weiterer These, dass dieses Denken seinen Rückhalt schon Platons (esoterischem) Philosophieren selbst findet [...], kaum vorbehaltlos zustimmen können, denn hier wird wieder auf den Kopf gestellt, was sich als Einsicht in die Ordnung von theoretischer und praktischer Philosophie für Platons (exoterisches) Philosophieren im allgemeinen nachweisen lässt. Jener von Krämer und Gaiser schon als Platonisch bezeichnete ,innerakademische` Entwurf überragt zwar alles Schriftliche, das wir von Platon besitzen, an spekulativer Geschlossenheit, lässt aber umgekehrt jene methodische Durchsichtigkeit vermissen, die [...] gerade die Höhe des platonischen Philosophierens ausmacht. [...] Wer darum auch die antike Geschichte des Platonismus über Xenokrates und Speusipp hinaus unmittelbar auf Platons authentische Lehrmeinung zurückführen will, der muss zunächst einmal die philosophische Glaubwürdigkeit der ,geschriebenen ${ }^{*}$ Platonischen Lehre, sofern sie jener , ungeschriebenen' Lehre nicht entspricht, entkräften. So lange dies nicht gelingt, bleibt dieser Platonismus von Speusipp bis hin zu Plotin in seiner spekulativen Gestalt ein Stück Metaphysik, dessen Verfasser gewissermaßen in der falschen Vorlesung Platons gesessen haben“ (Mittelstraß, 1970, S. 71).

${ }^{18}$ In Beckers veröffentlichten Arbeiten, aber auch im Nachlass, soweit er im Konstanzer Philosophischen Archiv erhalten ist, findet sich kein Hinweis darauf, dass Becker die „Tübinger Schule" noch zur Kenntnis genommen hätte.

${ }^{19}$ Vgl. Mittelstraß, 1967, besonders S. 34ff; Mittelstraß, 1970, S. $70 \mathrm{f}$ 
Gegen die hier angesprochene ,hellenistische Desorientierung der Vernunft", die in der neuplatonischen Platondeutung sichtbar werde, macht Mittelstraf (1970, S. 65ff.) darauf aufmerksam, dass es Platon wie Aristoteles um die Begründung „vernünftiger Selbständigkeit“ gehe, und dass im Zentrum ihres Denkens auch die Reflexion auf die „methodischen Voraussetzungen“ jener Selbständigkeit stehe (Mittelstraß, 1970, S. 50).

Obwohl Becker sich verbal zur metaphysischen Interpretation des Parmenides bekennt, finden wir bei ihm der Sache nach merkwürdigerweise nichts wesentlich Anderes als eine Ausarbeitung jener von Mittelstraß angesprochenen methodologischen Reflexion. Freilich handelt es sich dabei um eine methodologische Reflexion auf die Entstehung metaphysischer Aussagen über die Ordnung des Seienden bei Platon.

Becker ist im Übrigen davon überzeugt, dass genau dies den platonischen Texten gerecht werde. In seinem Aufsatz „Der pythagoreische Gedanke“ lieg im sehr daran, Platon vom Pythagoreismus abzurücken - trotz aller inhaltlichen Nähe. Der Grund dafür ist methodologisch: pythagoreisches Denken ist - ungeachtet der Faszination seiner Resultate - für Platon unmethodologische Spekulation: „Man darf aber auch Platon nicht völlig als Pythagoreer auffassen; denn er ist und bleibt, auch in der Spätzeit, ein kritischer Philosoph" (Becker, $1959,14){ }^{20}$ Und genau darauf, auf die methodenkritische Reflexion Platons, sind Beckers Ausführungen zu Platon in der Regel konzentriert. Beckers besondere Leistung besteht darin, in Platons Philosophie wieder jene mathematischen Methoden herausgearbeitet zu haben, die ihren zentralen methodologischen Antrieb bilden. Zweifellos am Bekanntesten ist Beckers heute weithin akzeptierte Rekonstruktion der Ideenzahlenlehre (Becker, 1957). In unserer Abhandlung zum Parmenides hingegen sind Proportional- und Symmetrieüberlegungen leitend. Mutatis mutandis kann auch aus Beckers Sicht gelten, was Mittelstraß (1970, S. 37) am Beispiel der Geometrie über die methodische Ordnung in Platons Denken sagt: „Vieles spricht [...] dafür, dass die gesamte Konzeption der (klassischen) Ideenlehre bereits [...] (einer) sehr speziellen Einsicht im geometrischen Bereich ${ }^{2 !}$ folgt und nicht etwa umgekehrt diese Einsicht erst ermöglicht hat."

Zurück zur ungeschriebenen Lehre. Beckers Stellung dazu entspricht voll und ganz seiner nüchternen methodologischen Orientierung. Obwohl er

${ }^{20}$ Als Beleg für diese These führt Becker unter anderem die Unterscheidung von Wahrheit und Mythos im Timaios sowie den auf der Unterscheidung von Modell und Wirklichkeit beruhenden idealen Charakter der Astronomie an. Mittelstraß (1962, S. 117 ff.) unterstreicht im Übrigen diese ideale Konzeption, indem er nachweist, dass das empirieorientierte Programm einer „Retder Phänomene", d h die Erklärung der beobachteten astronomischen Verhältnisse unter tung der Phan (S. 139).

(S. 139).
Gemeint ist die Unterscheidung von zeichnerisch realisierten geometrischen Gebilden und den

idealen Formen der Geometrie, welche die gezeichneten Gebilde darstellen sollen. gelegentlich das Wort „Geheimlehre“ verwendet, ist das, was er darüber zu sagen hat, eher das Dementi einer solchen.

Schon die Idee, aus dem zweiten Teil des Parmenides einen innerakademischen, „esoterischen" Lehrtext von Platons eigener Hand zu rekonstruieren, läuft der üblichen Auffassung zuwider, es handle sich bei der ungeschriebenen Lehre um schriftlich gar nicht auszudrückende Arcana, die von der geschriebenen Lehre grundverschieden, ,"wertvoller" ${ }^{\star 22}$ als diese und eben nur wenigen Eingeweihten zugänglich seien. ${ }^{23}$

Becker gibt mit seiner Rekonstruktion des zweiten Teils des Parmenides der ungeschriebenen Lehre eine unaufgeregte Deutung, die nirgendwo etwas ,hineingeheimnist". Der Gedanke, einen akademischen Schultext zu rekonstruieren, scheint mir im Übrigen sehr gut zu Wielands (1999) Deutung zu passen, wonach Platons Schriftkritik im Kern darauf hinauslaufe, dass die allem propositionalen Wissen zugrunde liegenden Inhalte nicht-propositionalen Wissens nicht in Aussagen (und damit Texten), d.h. propositional darstellbar seien. ${ }^{24}$ Damit ist freilich in Wielands (1999, S. 17) Augen ,keineswegs eine pauschale Entwertung alles Schriftlichen bezweckt". Es gehe Platon vielmehr darum, vor dem "Irrglauben" zu warnen, mit einem Text ,auch das bereits zu wissen und zu verstehen, wovon er handelt". Er ist nur ein „Werkzeug", das den für ein Verständnis notwendigen „Realkontext“ nicht darstellen kann. ${ }^{25}$ „Wissen lässt sich [...] nicht in der Weise mitteilen, in der Dinge übergeben oder ausgetauscht werden" (Wieland, 1999, S. 21). Und weiter: „Die Wirkung des geschriebenen Wortes kann von seinem Urheber nicht in gleicher Weise wie die des gesprochenen Wortes kontrolliert werden. [...] Erst die Kompetenz zur Explikation und zur Verteidigung aber zeigt das Vorliegen wirklichen Wissens an."

Unter diesen Prämissen lässt sich die Beckersche Rekonstruktion als gut platonisch verstehen: Platon schreibt für seinen Unterricht einen Text, der erst im realen Frage- und Antwortspiel der Schule über deiktische Mitteilungen und kontrollierte eigene Erfahrung des Schülers wirkliches Wissen zu vermitteln imstande ist.

Gibt es noch andere platonische Texte, die sich als innerschulische Übungsschriften verstehen lassen? Becker verneint diese Frage. Die zusammenhängenden Prosastücke der Briefe (nicht zu reden von den Problemen ihrer Echtheit)

${ }^{22}$ So Platon selbst (278d) in seiner Schriftkritik am Schluss des Phaidros.

${ }^{23}$ Wieland $(1999, \$ 2)$ liefert eine umfassende und überzeugende Kritik an der „Tübinger" Rekonstruktion der ungeschriebenen Lehre. Nicht zuletzt falle an ihr auf, dass die ,mit einem grossen Aufwand an Scharfsinn erzielten Resultate [...] inhaltlich nun einmal von extremer Dürftigkeit und Simplizität" seien (S. 43).

${ }^{24}$ Wieland $(1999,230)$ zählt hierzu „Fähigkeiten, Fertigkeiten, Kompetenzen und bewusstes Können, Urteilskraft, Gebrauchswissen und Erfahrung“. Er bemerkt weiter: „Durch alle diese Gestalten des Wissens wird ihrem Inhaber Welt und Wirklichkeit erschlossen. Über alle kann mit trilfe ven Ausen wird Inhalt keiner dieser Wissen formen lässt sich in Gestalt von Aussagen präsentieren und einem anderen mitteilen. "

${ }^{25}$ Die Dialogform kann als ein fiktiver Realkontext aufgefasst werden. 
seien sowieso an externe Empfänger gerichtet. Die Apologie sei kein Lehrstück, sondern solle einen Bericht liefern, der Timaios „,behält immer die Art eines weitausgesponnenen Mythos“" (Becker AII, S. 22f.) usw. ${ }^{26}$

„Vergleichbar müsste dagegen die verlorene Vorlesung ,über das Gute' sein, die aber leider nicht mehr in einer einigermaßen zuverlässigen Fassung zu rekonstruieren ist. Denn die bei Sextus Empiricus, Alexander von Aphrodisias, Simplicius u.a. erhaltenen angeblichen Fragmente sind im günstigsten Fall aristotelisch, aus der ,Nachschrift', in Wahrheit der Bearbeitung der platonischen Vorlesung durch Aristoteles stammend" (Becker, AII, S. 23).

Das ist ein wenig ermutigender Hinweis für die kämpferischen Verfechter der ungeschriebenen Lehre: sie rekonstruieren allenfalls des Aristoteles Auffassungen von Platons Vorlesung „Über das Gute“. ${ }^{27}$ Becker schliesst (AII, S. 23) mit einem für ein angemessenes Verständnis Platons extrem wichtigen, aber gelegentlich nicht beachteten Hinweis darauf, dass das, was „man gemeinhin als Lehre Platons bezeichnet, [...] allenfalls das Ergebnis von Rekonstruktionsversuchen auf der Grundlage dieses Werks ${ }^{\text {“28 }}$ darstellt und dass der „wirkliche“ Platon auf eine merkwürdige Weise verborgen bleibt:

"Aber selbst wenn eine wortgetreue Nachschrift der platonischen Ausführungen auf uns gekommen wäre, würde man vielleicht erkennen müssen, dass Ihre Ähnlichkeit mit der Urfassung des zweiten Teils des Parmenides nicht allzu gross ist. Denn aller Wahrscheinlichkeit nach waren die Erörterungen über das Gute schlicht dogmatische Darlegungen ohne den für den Parmenides so kennzeichnenden dialektischen Einschlag. So ,pythagoreisch ${ }^{\prime} \pi \varepsilon p \grave{~} \tau \alpha \dot{\gamma} \gamma \alpha 0 \mathrm{ov}$ gewesen sein dürfte, so ,eleatisch' ist die Urfassung des Parmenides. Beide aber sind ,Masken des dionysischen Philosophen, der so selten im eigenen Namen redet."

\section{5. „Vorschrift" und „Urfassung“}

Im zweiten Abschnitt des ersten Teils seiner Abhandlung erörtert Becker ausführlich die Aufgaben, die sich einer nicht willkürlichen Elimination der Dialogform stellen. Der Anfang ist einfach: „Es müssen die - nichtssagenden Antworten des Aristoteles im Gespräch sämtlich gestrichen werden. Das ist in

${ }^{26}$ Merkwürdigerweise zieht Becker den Sophistes nicht als weiteres mögliches Beispiel einer „, dialogisierten" Lehrschrift in Betracht. Die dialogische Rolle des Theaitetos im Sophistes scheint
sich auf den ersten Blick nicht allzu sehr von der des Aristoteles im Parmenides zu unterscheisich auf den ersten Blick nicht allzu sehr von der des Aristoteles im Parmenides zu unterscheiden.

${ }^{27}$ Wie sehr eingebettet ins eigene Philosophieren und wie wenig verlässlich damit die „Philosophiegeschichte" des Aristoteles ist, hat seit den 30er Jahren bereits Harold Cherniss gezeigt. Neuerdings hat L. Zhmud (1997) überzeugend nachgewiesen, dass die allgemein Pythagoras und dem frühen Pythagoreismus zugeschriebene "Zahlenmetaphysik" einzig und allein auf dem Zeugnis des Aristoteles beruht. Nach den weniger ,interessierten“Q Quellen ist sie hingegen mehr als unwahrscheinlich.

${ }^{28}$ Wieland $(1999$, S. 50) der Tat ausführbar [...] ohne dass ein einziger Gedanke verloren geht, denn ein solcher ist in den Äusserungen des Aristoteles nicht enthalten" (AII, 4). ${ }^{29} \mathrm{Nach}$ einer zweiten Regel sind rhetorische Fragesätze in ,sinngleiche gewöhnliche Aussagesätze zurückzuverwandeln und das ohne Willkür". Eine andere befasst sich mit rhetorischen Fragen, die eine negative Antwort erwarten, die dann natürlich die Einfügung einer Negation in der betreffenden Aussage verlangen, wobei freilich nicht immer festgelegt ist, wo sie einfügt werden soll oder welche der beiden griechischen Formen der Verneinung anzuwenden ist. Nicht frei von einer gewissen Willkür ist die Behandlung von Doppelfragen oder auch der Frage ,wieso?“ $(\pi \hat{\omega} \zeta ;)$. Im letzteren Fall hat, die Antwort des Parmenides einen begründenden Sinn, der bei der Umwandlung in fortlaufende Rede nicht verloren gehen darf und etwa durch ein an geeigneter Stelle eingeschobenes $\gamma$ óp erhalten werden muss" (AII, 5).

Der zweite Teil der Beckerschen Abhandlung (,Vorschrift für die Umwandlung der Dialogform in die Urfassung") enthält nun nichts anderes als eine Auflistung aller Stellen des zweiten Teils des Parmenides, an denen Dialogelemente eliminiert wurden. Der dritte Teil schliesslich besteht in einer (vollständigen), ${ }^{30}$ säuberlich von Hand ausgeführten Reinschrift der auf die oben angegebene Weise rekonstruierten „Urfassung" des zweiten Teils des Parmenides (137c4 bis zum Schluss).

Es ist zu wünschen, dass mit der geplanten Veröffentlichung des Beckerschen Nachlasses, die Diskussion um die ungeschriebene Lehre angesichts eines - wenn Becker Recht hat - ,geschriebenen“ Stücks neue Perspektiven erhalten wird. ${ }^{31}$ Es könnte ja beispielsweise so sein, dass Platon darauf verzichten wollte, besonders komplizierte Texte (und der zweite Teil des Parmenides ist gewiss hochkompliziert) an eine Öffentlichkeit zu bringen, die sie ohnehin nicht verstanden und sich daher - zum Schaden der weithin verständlichen Texte eher darüber mokiert hätte. Vielleicht wird sich einmal ergeben, dass die Unterschiede zwischen der exoterischen und esoterischen Lehre Platons nicht viel tiefgreifender sind als diejenigen, die in den zwanziger Jahren des vorigen Jahrhunderts zwischen den, nur Spezialisten zugänglichen, hochprofessionellen und technischen Diskussionen in der beginnenden analytischen Philosophie und Wissenschaftstheorie im „Wiener Kreis" einerseits und deren popularisierender Verbreitung in den Schriften und öffentlichen Vorträgen des „Vereins Ernst Mach" andererseits bestanden haben.

${ }^{29}$ Schudoma (2001, S. 46) freilich glaubt resümieren zu können, „dass die kurzen Antworten des Aristoteles nicht ganz bedeutungslos sind“".

${ }^{30}$ Merkwürdigerweise hat Becker im zweiten Abschnitt des ersten Teils (AII, S. 6) den Satz stehen gelassen: „Aus Raumgründen kann hier nicht der ganze Text des zweiten Teils des Parmenides umgeschrieben vorgelegt werden".

"Die geplante Herausgabe steht unter der Federführung von Annemarie Gethmann-Siefert (Ha- 
Becker, Oskar: 1957, ,Zum Problem der platonischen Idealzahlen (eine Retraktation) “, in: ders., Zwei Untersuchungen zur antiken Logik, Wiesbaden: Otto Harrasowitz, S. $1-22$.

Becker, Oskar, 1959, Größe und Grenze der mathematischen Denkweise, Freiburg/München: Karl Alber.

Becker, Oskar, 1963, Dasein und Dawesen: Gesammelte philosophische Aufsätze, Pfullingen: Neske.

Brisson, Luc (Hg.), 1994, Parménide, traduction inédite, introduction et notes, Paris: GF-Flammarion.

Gaiser, Konrad, 1968, Platons ungeschriebene Lehre. Studien zur systematischen und geschichtlichen Begründung der Wissenschaften in der Platonischen Schule, Stuttgart: Ernst Klett, 2. Aufl. (1. Auflage 1963).

Gonzalez, Franciso J. (Hg.), 1995, The Third Way. New Directions in Platonic Studies, London: Rowman \& Littlefield.

Guthrie, W. K. C., 1978, A History of Greek Philosophy, Vol. V (The Later Plato and the Academy), Cambridge: University Press.

Kutschera, Franz von, 1995, Platons ,Parmenides‘, Berlin/New York: Walter de Gruyter.

Meinwald, Constance C., 1991, Plato's Parmenides, New York/Oxford: Oxford University Press,

Mittelstraß, Jürgen, 1962, Die Rettung der Phänomene. Ursprung und Geschichte eines antiken Forschungsprinzips, Berlin: Walter de Gruyter.

Mittelstraß, Jürgen, 1967, „Ontologia more geometrico demonstrata“, in: Philosophische Rundschau, 14, S. 27-40.

Mittelstraß, Jürgen, 1970, Neuzeit und Aufklärung. Studien zur Entstehung der neuzeitlichen Wissenschaft und Philosophie, Berlin/New York: Walter de Gruyter.

Schudoma, Ingeborg, 2001, Platons Parmenides. Kommentar und Deutung, Würzburg: Königshausen und Neumann.

Vegetti, Mario, 2003, ,, Solo Platone non c'era' “, Paradigmi, 62, S. 261-277.

Wieland, Wolfgang, 1999, Platon und die Formen des Wissens, Göttingen: Vandenhoeck \& Ruprecht (2., durchgesehene und um einen Anhang und ein Nachwort erweiterte Auflage. Erstauflage 1982)

Wolters, Gereon, 2002, „Philosophie im Nationalsozialismus: der Fall Oskar Becker", in: Jürgen Mittelstraß/Annemarie Gethmann-Siefert (Hg.), Die Philosophie und die Wissenschaften. Zum Werk Oskar Beckers, München: W. Fink, 27-64.

Wolters, Gereon/ Schroeder(-Heister), Peter, 1979, Der wissenschaftliche Nachlass von Hugo Dingler (1881-1954) - Verzeichnis - mit einer Bibliographie der Schriften Dinglers, Konstanz: Universität (als Manuskript gedruckt).

Wundt, Max, 1935, Platons Parmenides, Stuttgart/Berlin: W. Kohlhammer.

Zhmud', Leonid, 1997, Wissenschaft, Philosophie und Religion im frühen Pythagoreismus, Berlin: Akademie Verlag. 